EPJ manuscript No.

(will be inserted by the editor)

\title{
Influence of Chaos on the fusion enhancement by electron screening
}

\author{
S. Kimura ${ }^{1}$, A. Bonasera ${ }^{1}$ and S. Cavallaro ${ }^{12}$ \\ 1 Laboratorio Nazionale del Sud, INFN, via Santa Sofia, 62, 95123 Catania, Italy \\ ' 2 Dipartimento di Fisica, Università degli Studi di Catania, via Santa Sofia, 64, 95123 Catania, Italy \\ Received: date / Revised version: date
}

\begin{abstract}
We study the effect of screening by bound electrons in low energy nuclear reactions. We use molecular dynamics to simulate the reactions involving many electrons: $\mathrm{D}+d, \mathrm{D}+\mathrm{D},{ }^{3} \mathrm{He}+d,{ }^{3} \mathrm{He}+\mathrm{D},{ }^{6} \mathrm{Li}+d$, ${ }^{6} \mathrm{Li}+\mathrm{D},{ }^{7} \mathrm{Li}+p,{ }^{7} \mathrm{Li}+\mathrm{H}$. Quantum effects corresponding to the Pauli and Heisenberg principles are enforced by constraints in terms of the phase space occupancy. In addition to the well known adiabatic and sudden limits, we propose a new "dissipative limit" which is expected to be important not only at high energies but in the extremely low energy region. The dissipative limit is associated with the chaotic behavior of the electronic motion. It affects also the magnitude of the enhancement factor. We discuss also numerical experiments using polarized targets. The derived enhancement factors in our simulation are in agreement with those extracted within the $R$-matrix approach.
\end{abstract}

PACS. 25.45.- $\mathrm{z}-34.10 .+\mathrm{x}$

\section{Introduction}

The relation between the tunneling process and dynamical chaos has been discussed with great interests in recent years. [1,2] Though the tunneling is completely quantum mechanical phenomenon, it is influenced by classical chaos. In the sense that the the chaos causes the fluctuation of the classical action which essentially determines the tunneling probability. We study the phenomenon by examining the screening effect by bound electrons in the low energy fusion reaction. In the low energy region the experimental cross sections with gas targets show an increasing enhancement with decreasing bombarding energy with respect to the values obtained by extrapolating from the data at high energies [3]. Many studies attempted to attribute the enhancement of the reaction rate to the screening effects by bound target electrons. In this context one often estimates the screening potential as a constant decrease of the barrier height in the tunneling region through a fit to the data. A puzzle has been that the screening potential obtained by this procedure exceeds the value of the so called adiabatic limit, which is given by the difference of the binding energies of the united atoms and of the target atom and it is theoretically thought to provide the maximum screening potential [4]. Over these several years, the redetermination of the bare cross sections has been proposed theoretically [5] and experimentally [6], using the Trojan Horse Method [7,8,21]. The comparison between newly obtained bare cross sections, i.e., astrophysical Sfactors, and the cross sections by the direct measurements gives a variety of values for the screening potential. These values are often smaller than the sudden limit or larger than the adiabatic limit. Theoretical studies performed using the time-dependent Hartree-Fock(TDHF) scheme 9, 10] suggest that the screening potential is between the sudden and the adiabatic limits.

One of the aims of this paper is to try to assess the effect of the screening quantitatively. Up to now, the dynamical effects of bound electrons have been studied only in some limited cases with a few bound electrons (the $\mathrm{D}+d$ with atomic target 9, 10 and molecular $\mathrm{D}_{2}$ target [1], the ${ }^{3} \mathrm{He}+d[9]$ ) with the TDHF method. We investigate here the dynamical effects, including the tunneling region, for other systems with many bound electrons; $\mathrm{D}+\mathrm{D},{ }^{3} \mathrm{He}+\mathrm{D}$, looking the effect of the electron capture of projectile. We see also some reactions including $\mathrm{Li}$ isotopes; ${ }^{6} \mathrm{Li}+d$, ${ }^{6} \mathrm{Li}+\mathrm{D},{ }^{7} \mathrm{Li}+p$ and ${ }^{7} \mathrm{Li}+\mathrm{H}$.

To simulate the effects of many electrons, we use the constrained molecular dynamics (CoMD) model [2 12, 15]. At very low energies fluctuations are anticipated to play a substantial role. Such fluctuations are beyond the TDHF scheme. Not only TDHF calculations are, by construction, cylindrically symmetric around the beam axis. Such a limitation is not necessarily true in nature and the mean field dynamics could be not correct especially in presence of large fluctuations. Molecular dynamics contains all possible correlations and fluctuations due to the initial conditions(events). For the purpose of treating quantummechanical systems like target atoms and molecules, we 
use classical equations of motion with constraints to satisfy the Heisenberg uncertainty principle and the Pauli exclusion principle for each event 12. In extending the study to the lower incident energies, we would like to stress the connection between the motion of bound electrons and chaos. In fact, depending on the dynamics, the behavior of the electron(s) is unstable and influences the relative motion of the projectile and the target. The feature is caused by the nonintegrablility of the $N$-body $\operatorname{system}(N \geq 3)$ and it is well known that the tunneling probability can be modified by the existence of chaotic environment. We discuss the enhancement factor of the laboratory cross section in connection with the integrability of the system by looking the inter-nuclear and electronic oscillational motion. More specifically we analyze the frequency shift of the target electron due to the projectile and the small oscillational motion induced by the electron to the relative motion between the target and the projectile. We show that the increase of chaoticity in the electron motion decreases the fusion probability.

The paper is organized as follows. In sect. 2 we introduce the enhancement factor $f_{e}$ and describe the essence of the Constrained molecular dynamics approach briefly. In sect. [3 we apply it to asses the effect of the bound electrons during the nuclear reactions. We discuss also the relation between the amplitudes of the inter-nuclear oscillational motion and the enhancement factor. We summarize the paper in sect. [4

\section{Formalism}

\subsection{Enhancement Factor}

We denote the reaction cross section at incident energy in the center of mass $E$ by $\sigma(E)$ and the cross section obtained in absence of electrons by $\sigma_{0}(E)$. The enhancement factor $f_{e}$ is defined as

$$
f_{e} \equiv \frac{\sigma(E)}{\sigma_{0}(E)} .
$$

If the effect of the electrons is well represented by the constant shift $U_{e}$ of the potential barrier, following [13], $\left(U_{e} \ll E\right)$ :

$$
f_{e} \sim \exp \left[\pi \eta(E) \frac{U_{e}}{E}\right]
$$

where $\eta(E)$ is the Sommerfeld parameter [14].

\subsection{Constrained Molecular Dynamics}

We estimate the enhancement factor $f_{e}$ numerically using molecular dynamics approach;

$$
\frac{d \mathbf{r}_{i}}{d t}=\frac{\mathbf{p}_{i} c^{2}}{\mathcal{E}_{i}}, \quad \frac{d \mathbf{p}_{i}}{d t}=-\nabla_{\mathbf{r}} U\left(\mathbf{r}_{i}\right),
$$

where $\left(\mathbf{r}_{i}, \mathbf{p}_{i}\right)$ are the position, momentum of the particle $i$ at time $t . \mathcal{E}_{i}=\sqrt{\mathbf{p}_{i}^{2} c^{2}+m_{i}^{2} c^{4}}, U\left(\mathbf{r}_{i}\right)$ and $m_{i}$ are its energy, Coulomb potential and mass, respectively. We set the starting point of the reaction at $10 \AA$ inter-nuclear separation. In Eqs. (3) we do not take into account the quantum effect of Pauli exclusion principle and Heisenberg principle. As it is well known that these classical equations (3) can be derived by using the variational calculus of Lagrangian $\mathcal{L}$ of the classical system as well. So as to take the feature of the Pauli blocking into account in this framework, we use the Lagrange multiplier method for constraints.

Our constraints which correspond to the Pauli blocking is $\bar{f}_{i} \leq 1$ in terms of phase space density, note that the phase space density can be directly related to the distance of two particles, i.e., $\mathbf{r}_{i j} \mathbf{p}_{i j}$, in the phase space. Here $\mathbf{r}_{i j}=$ $\left|\mathbf{r}_{i}-\mathbf{r}_{j}\right|$ and $\mathbf{p}_{i j}=\left|\mathbf{p}_{i}-\mathbf{p}_{j}\right|$. The relation $\bar{f}_{i} \leq 1$ is fulfilled, if $\mathbf{r}_{i j} \mathbf{p}_{i j} \geq \xi_{P} \hbar \delta_{S_{i}, S_{j}}$, where $\xi_{P}=2 \pi(3 / 4 \pi)^{2 / 3} . i, j$ refer only to electrons and $S_{i}, S_{j}(= \pm 1 / 2)$ are their spin projection. For the Heisenberg principle $\mathbf{r}_{i j} \mathbf{p}_{i j} \geq \xi_{H} \hbar$, where $\xi_{H}=1, i$ and $j$ refer to not only electrons but the nucleus. It is determined to reproduce the correct energy of hydrogenic atoms. Obviously the conditions $\mathbf{r}_{i j} \mathbf{p}_{i j}=\xi_{H(P)} \hbar$ must be fulfilled in the ground state configuration rather than $\mathbf{r}_{i j} \mathbf{p}_{i j}>\xi_{H(P)} \hbar$.

Using these constraints, the Lagrangian of the system can be written down as

$$
\begin{aligned}
\mathcal{L} & =\sum_{i} \frac{\mathbf{p}_{i}^{2} c^{2}}{\mathcal{E}_{i}}-\sum_{i, j(\neq i)} U\left(\mathbf{r}_{i j}\right)+\sum_{i, j(\neq i)} \lambda_{i}^{H}\left(\frac{\mathbf{r}_{i j} \mathbf{p}_{i j}}{\hbar}-1\right) \\
& +\sum_{i, j(\neq i)} \lambda_{i}^{P}\left(\frac{\mathbf{r}_{i j} \mathbf{p}_{i j}}{\xi_{P} \hbar} \delta_{S_{i}, S_{j}}-1\right)
\end{aligned}
$$

where $\lambda_{i}^{P}$ and $\lambda_{i}^{H}$ are Lagrange multipliers. The variational calculus leads

$$
\begin{aligned}
\frac{d \mathbf{r}_{i}}{d t} & =\frac{\mathbf{p}_{i} c^{2}}{\mathcal{E}_{i}}+\frac{1}{\hbar} \sum_{j(\neq i)}\left(\frac{\lambda_{i}^{H}}{\xi_{H}}+\frac{\lambda_{i}^{P}}{\xi_{P}} \delta_{S_{i}, S_{j}}\right) \mathbf{r}_{i j} \frac{\partial \mathbf{p}_{i j}}{\partial \mathbf{p}_{i}} \\
\frac{d \mathbf{p}_{i}}{d t} & =-\nabla_{\mathbf{r}} U\left(\mathbf{r}_{i}\right)-\frac{1}{\hbar} \sum_{j(\neq i)}\left(\frac{\lambda_{i}^{H}}{\xi_{H}}+\frac{\lambda_{i}^{P}}{\xi_{P}} \delta_{S_{i}, S_{j}}\right) \mathbf{p}_{i j} \frac{\partial \mathbf{r}_{i j}}{\partial \mathbf{r}_{i}}(6)
\end{aligned}
$$

In order to obtain the atomic ground-state configuration, We perform the time integration of the eqs. (5) and (6). The value of $\lambda_{i}^{H}$ and $\lambda_{i}^{P}$ are determined depending on the magnitude of $\mathbf{r}_{i j} \mathbf{p}_{i j}$. If $\mathbf{r}_{i j} \mathbf{p}_{i j}$ is (smaller)larger than $\xi_{H(P)} \hbar, \lambda$ has positive(negative) sign. Thus we change the phase space occupancy of the system. The constraints restrict us to variations $\Delta \mathcal{L}=0$ that keep the constraints always true [15]. In this way we obtain many initial conditions which occupy different points in the phase space microscopically.

In order to treat the tunneling process, we define the collective coordinates $\mathbf{R}^{\text {coll }}$ and the collective momentum $\mathbf{P}^{\text {coll }}$ as

$$
\mathbf{R}^{\text {coll }} \equiv \mathbf{r}_{P}-\mathbf{r}_{T} ; \quad \mathbf{P}^{\text {coll }} \equiv \mathbf{p}_{P}-\mathbf{p}_{T}
$$

where $\mathbf{r}_{T}, \mathbf{r}_{P}\left(\mathbf{p}_{T}, \mathbf{p}_{P}\right)$ are the coordinates(momenta) of the target and the projectile nuclei, respectively. When 
the collective momentum becomes zero, we switch on the collective force, which is determined by $\mathbf{F}_{P}^{\text {coll }} \equiv \dot{\mathbf{P}}^{\text {coll }}$ and $\mathbf{F}_{T}^{\text {coll }} \equiv-\dot{\mathbf{P}}^{\text {coll }}$, to enter into imaginary time 16. We follow the time evolution in the tunneling region using the equations,

$$
\frac{d \mathbf{r}_{T(P)}^{\Im}}{d \tau}=\frac{\mathbf{p}_{T(P)}^{\Im}}{\mathcal{E}_{T(P)}} ; \quad \frac{d \mathbf{p}_{T(P)}^{\Im}}{d \tau}=-\nabla_{\mathbf{r}} U\left(\mathbf{r}_{T(P)}^{\Im}\right)-2 \mathbf{F}_{T(P)}^{c o l l},
$$

where $\tau$ is used for imaginary time to be distinguished from real time $t . \mathbf{r}_{T(P)}^{\Im}$ and $\mathbf{p}_{T(P)}^{\Im}$ are position and momentum of the target (the projectile) during the tunneling process respectively. Adding the collective force corresponds to inverting the potential barrier which becomes attractive in the imaginary times. The penetrability of the barrier is given by [16]

$$
\Pi(E)=(1+\exp (2 \mathcal{A}(E) / \hbar))^{-1},
$$

where the action integral $\mathcal{A}(E)$ is

$$
\mathcal{A}(E)=\int_{r_{b}}^{r_{a}} \mathbf{P}^{\text {coll }} d \mathbf{R}^{\text {coll }}
$$

$r_{a}$ and $r_{b}$ are the classical turning points. The internal classical turning point $r_{b}$ is determined using the sum of the radii of the target and projectile nuclei. Similarly from the simulation without electron, we obtain the penetrability of the bare Coulomb barrier $\Pi_{0}(E)$.

Since nuclear reaction occurs with small impact parameters on the atomic scale, we consider only head on collisions. The enhancement factor is thus given by eq. (11),

$$
f_{e}=\Pi(E) / \Pi_{0}(E)
$$

for each event in our simulation. Thus we have an ensemble of $f_{e}$ values at each incident energy.

\section{Application to the Electron Screening Problem}

\subsection{1 $\mathrm{D}+d$ and $\mathrm{D}+\mathrm{D}$ reactions}

Fig. 1 shows the incident energy dependence of the enhancement factor for the reactions $\mathrm{D}+d$ and $\mathrm{D}+\mathrm{D}$, where the systems involve 1 and 2 electrons respectively. The open and closed squares show the average enhancement factors $\bar{f}_{e}$ over events for the reactions $\mathrm{D}+d$ and $\mathrm{D}+\mathrm{D}$, respectively. The variances $\Sigma=\sqrt{\bar{f}_{e}^{2}-\left(\bar{f}_{e}\right)^{2}}$ are shown with error bars. The dotted and dash-dotted curves show the enhancement factors in the adiabatic limit $f_{e}^{(A D)}$ for an atomic deuterium target and it is obtained by assuming equally weighted linear combination of the lowest-energy gerade and ungerade wave function for the electron, reflecting the symmetry in the $\mathrm{D}+d$, i.e.,

$$
f_{e}^{(A D)}=\frac{1}{2}\left(e^{\pi \eta(E) \frac{U_{e}^{(g)}}{E}}+e^{\pi \eta(E) \frac{U_{e}^{(u)}}{E}}\right),
$$

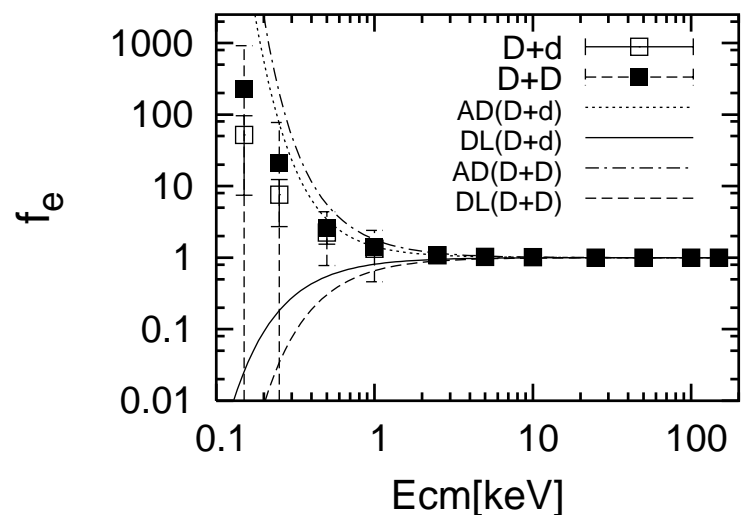

Fig. 1. Enhancement factor as a function of incident centerof-mass energy for the reactions $\mathrm{D}+d$ and $\mathrm{D}+\mathrm{D}$. Error-bars represent the variances obtained from the events generated for each beam energy.

where $U_{e}^{(g)}=40.7 \mathrm{eV}$ and $U_{e}^{(u)}=0.0 \mathrm{eV}$ 10, for $\mathrm{D}+d$ case. If we take into account the electron capture of the projectile, i.e., in the case of $\mathrm{D}+\mathrm{D}$, the enhancement factor in the adiabatic limit is

$$
f_{e}^{(A D)}=\frac{1}{4} e^{\pi \eta(E) \frac{U_{e}^{(g . s .)}}{E}}+\frac{3}{4} e^{\pi \eta(E) \frac{U_{e}^{(1 e s)}}{E}},
$$

where $U_{e}^{(\text {g.s. })}=51.7 \mathrm{eV}$ and $U_{e}^{(1 e s)}=31.9 \mathrm{eV}$ [17]. The solid curve and dashed curve show the enhancement factors in the dissipative limit $f_{e}^{(D L)}$ for the reactions $\mathrm{D}+d$ and $\mathrm{D}+\mathrm{D}$ respectively. Notice how the calculated enhancement factor with their variances nicely ends up between the adiabatic and the dissipative limits. We performed also a fit of our data using eq. (2) including the very low energy region and obtained $U_{e}=15.9 \pm 2.0 \mathrm{eV}$ for $\mathrm{D}+d$ case and $U_{e}=21.6 \pm 0.3 \mathrm{eV}$ for $\mathrm{D}+\mathrm{D}$.

Now we look at the oscillational motions of the particle's coordinates as the projection on the $z$-axis (the reaction axis). We denote the $z$-component of $\mathbf{r}_{T}, \mathbf{r}_{P}$ and $\mathbf{r}_{e}$ as $z_{T}, z_{P}$ and $z_{e}$, respectively. Practically, we examine the oscillational motion of the electron around the target $z_{T e}=z_{e}-z_{T}$ and the oscillational motion of the internuclear motion, i.e., the motion between the target and the projectile, $z_{s}=z_{T}+z_{P}$, which essentially would be zero due to the symmetry of the system in the absence of the perturbation. In Fig. 2 these two values are shown for 2 events, which have the enhancement factor $f_{e}=$ 170.8 (ev. A), and $f_{e}=6.5$ (ev. B), at the incident energy $E_{c m}=0.15 \mathrm{keV}$. The panels show the $z_{s}, z_{T e}$ as a function of time. The stars indicate the time at which the system reaches the classical turning point. It is clear that in the case of event B the orbit of the electron is much distorted from the unperturbed one than in event A. Characteristics of $z_{s}$ are that (1) its value often becomes zero, as it is expected in the un-perturbed system, and (2) the component of the deviation from zero shows periodical behavior. It is remarkable that the amplitude of the deviation becomes quite large at some points in the case of event B 

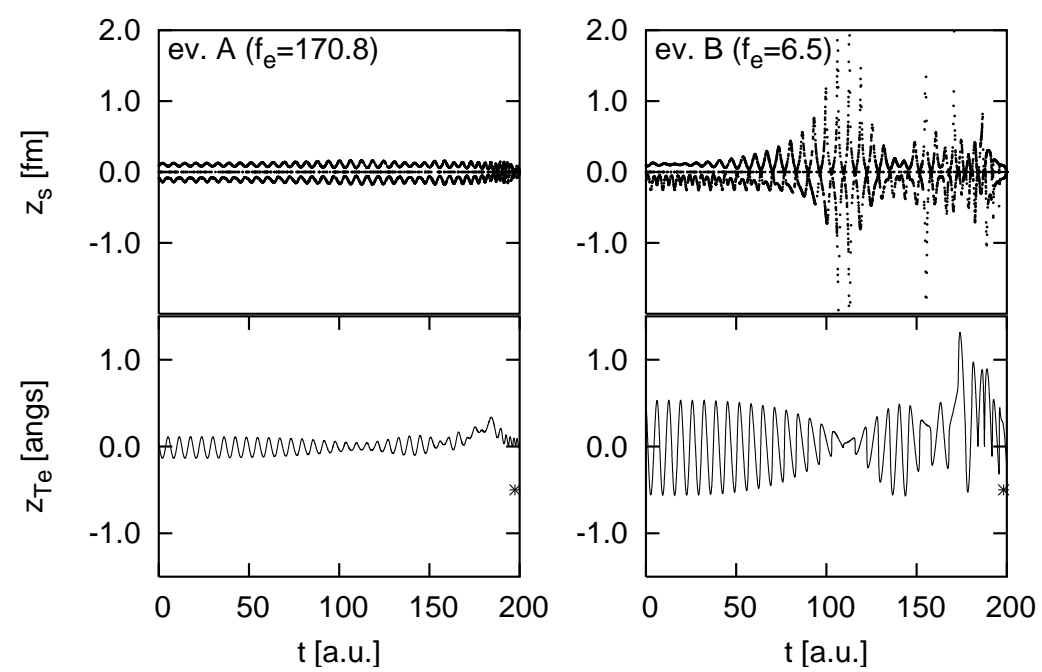

Fig. 2. The oscillational motion of the electron around the target (lower panels) and the inter-nuclear motion (upper panels) as a function of time, in atomic unit, for two events, with large $f_{e}\left(\right.$ ev. A) and small $f_{e}($ ev. B), for the D+d reaction at the incident energy $0.15 \mathrm{keV}$. The inter-nuclear separation is $10 \AA$ at $t=0$.

which shows the small enhancement factor. Note that in event B one observes clear beats, i.e., resonances. Thus for two events, with the same macroscopic initial conditions, we have a completely different outcome, which is a definite proof of chaos in our 3-body system. We can understand these results in first approximation by considering the motion of the ions to be much slower than the rapidly oscillating motion of the electrons. 2] From the Fig 2 we can deduce the following important fact. If the motion of the electron is initially in the plane perpendicular to the reaction axis, the enhancement factor is large, event $\mathrm{A}$ (notice $\left|z_{T e}\right| \ll R_{B}$, i.e., the Bohr radius, at $t \sim 0)$. On the other hand if there is a substantial projection of the electron motion, as in event $\mathrm{B}$ (the amplitude of $\left|z_{T e}\right| \sim R_{B}$ at $t \sim 0$ ), on the reaction axis the enhancement factor is relatively small because of the increase of chaoticity. The fact suggests that if one performs experiments at very low bombarding energies with polarized targets, the enhancement factor can be controlled by changing the polarization. The largest enhancement would be gained with targets polarized perpendicularly to the beam axis.

In order to test this estimation, we prepared ensembles of target atoms which are polarized perpendicular $\left(\mathrm{P}_{\perp}\right)$ and parallel $\left(\mathrm{P}_{\|}\right)$to the beam axis, numerically. In Fig. 3 we show the incident energy dependence of the average enhancement factor for the $\mathrm{P}_{\perp}$ and $\mathrm{P}_{\|}$targets with pluses and crosses, particularly in the low energy region. The enhancement factors from the $\mathrm{P}_{\perp}$ targets are always larger than that from the $\mathrm{P}_{\|}$targets. In contrast to the average enhancement from the $\mathrm{P}_{\perp}$ targets, which increases monotonically as the incident energy becomes smaller, the average enhancement from the $\mathrm{P}_{\|}$targets fluctuates. It has also large variances at low energies. Remarkable thing is that with the parallel targets the enhancement factor often becomes less than 1 . It means that in this case the

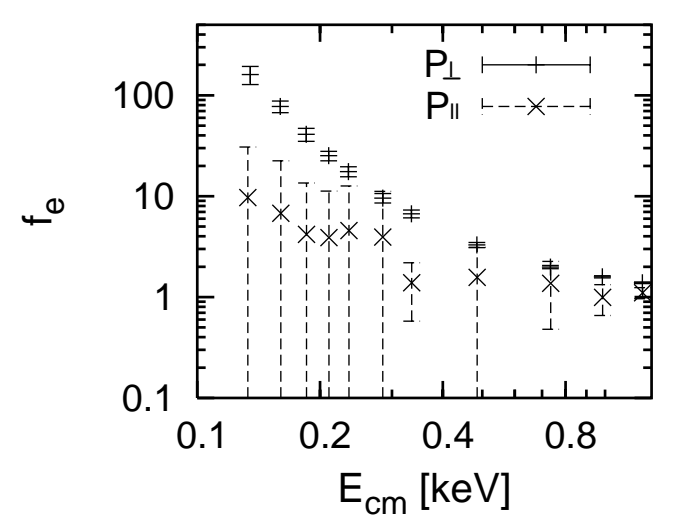

Fig. 3. Incident energy dependence of the enhancement factor for $\mathrm{P}_{\perp}$ and $\mathrm{P}_{\|}$targets.

bound electron gives the effect of hindrance to the tunneling probability.

\subsection{2 ${ }^{3} \mathrm{He}+d$ and ${ }^{3} \mathrm{He}+\mathrm{D}$ reactions}

An excess of the screening potential was reported for the reactions ${ }^{3} \mathrm{He}+d$ with atomic gas ${ }^{3} \mathrm{He}$ target, and $\mathrm{D}_{2}+$ ${ }^{3} \mathrm{He}$ with deuterium molecular gas target, for the first time in the reference 3. Since then various experiments have been performed for these reactions. The incident energy covers from $5 \mathrm{keV}$ to $50 \mathrm{keV}$ for ${ }^{3} \mathrm{He}+d$. Though once the problem of the discrepancy between experimental data and theoretical prediction seemed to be solved by considering the correct energy loss data 19, recent measurements using measured energy loss data [20] report larger screening potentials than in the adiabatic limit for both reactions. 
The electron capture by the projectile plays a minor role in the case of ${ }^{3} \mathrm{He}+\mathrm{d}$, since electrons are more bound in helium targets. However in the recent measurement Aliotta et al. was performed using molecular $\mathrm{D}_{2}^{+}$and $\mathrm{D}_{3}^{+}$ targets 20. Thus we assess the contribution from the reaction ${ }^{3} \mathrm{He}+\mathrm{D}$, as well.

The enhancement factor in the adiabatic limit give $U_{e}=119 \mathrm{eV}$ for ${ }^{3} \mathrm{He}+d$ and $U_{e}=110 \mathrm{eV}$ for ${ }^{3} \mathrm{He}+\mathrm{D}$, respectively. These are shown in the figure 4 with the solid curve for ${ }^{3} \mathrm{He}+d$ and with the dashed curve for ${ }^{3} \mathrm{He}+\mathrm{D}$. The comparison of these two adiabatic limits implies that the electron capture of projectile would give a hindrance compared with the bare deuteron projectile. Meanwhile the latest analysis of the experimental data using $R$-matrix two level fit 5] suggests the screening potential $U_{e}=60$ $\mathrm{eV}$ (corresponding enhancement factor is shown with dotted curve). The comparison between direct measurement and an indirect method, the Trojan Horse method, suggests the screening potential $U_{e}=180 \pm 40 \mathrm{eV}$ ( the corresponding enhancement factor is shown with dot-dashed curve) 21. The average enhancement factors $\bar{f}_{e}$ over events in our simulations using the CoMD are shown with the

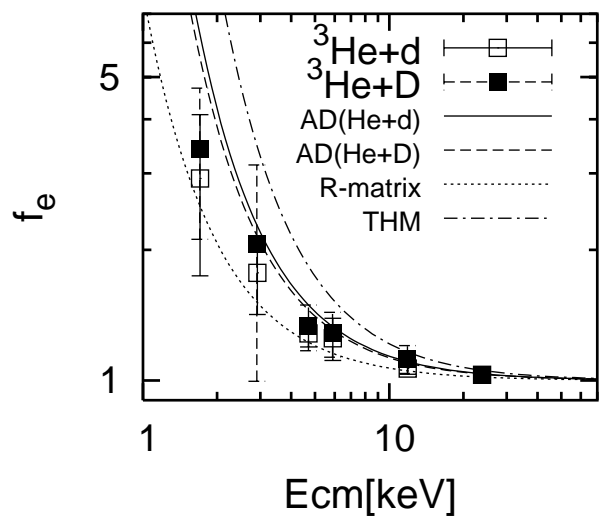

Fig. 4. Enhancement factor as a function of incident centerof-mass energy for the reactions ${ }^{3} \mathrm{He}+d$ and ${ }^{3} \mathrm{He}+\mathrm{D}$.

open and closed squares for the reactions ${ }^{3} \mathrm{He}+d$ and ${ }^{3} \mathrm{He}+\mathrm{D}$, respectively. The enhancement factors of the both reactions ${ }^{3} \mathrm{He}+d$ and ${ }^{3} \mathrm{He}+\mathrm{D}$ are in agreement with the extracted values using the $R$-matrix approach within the variances over all the events. Notice that our calculated enhancement factors for the two systems display an opposite trend as compared to the adiabatic limits. The average enhancement factor of the reaction ${ }^{3} \mathrm{He}+\mathrm{D}$ agrees with the estimation of the adiabatic limit and the reaction ${ }^{3} \mathrm{He}+\mathrm{d}$ is below the corresponding adiabatic limit. The paradoxical feature comes from the fact that an electron between the two ions is often kicked out during the reaction process, i.e., the electron configuration seldom settles down the ${ }^{5} \mathrm{Li}^{+}$ground state in the reaction ${ }^{3} \mathrm{He}+\mathrm{d}$. It is known as autoionization in the context of the Classical Trajectory Monte Carlo method 22. Instead in the case of the ${ }^{3} \mathrm{He}+\mathrm{D}$, the deuterium projectile brings its bound electron in a tight bound state around the unified nuclei of ${ }^{3} \mathrm{He}$ and $d$, practically it ends up with a ground state configuration of the ${ }^{5} \mathrm{Li}$ atom. The fits of the obtained enhancement factors suggests the screening potentials $U_{e}=82.4 \pm 1.9 \mathrm{eV}$ for the ${ }^{3} \mathrm{He}+d$ and $U_{e}=102.8 \pm 3.0 \mathrm{eV}$ for the ${ }^{3} \mathrm{He}+\mathrm{D}$.

\subsection{3 ${ }^{6} \mathrm{Li}+d,{ }^{6} \mathrm{Li}+\mathrm{D},{ }^{7} \mathrm{Li}+p$ and ${ }^{7} \mathrm{Li}+\mathrm{H}$}

The S-factors for the reactions ${ }^{6} \mathrm{Li}+d,{ }^{6} \mathrm{Li}+p$ and ${ }^{7} \mathrm{Li}+p$ were measured over the energy range $10 \mathrm{keV}<E_{c m}<500$ $\mathrm{keV}$ by Engstler, et al. 23. They used LiF solid targets and deuteron projectiles as well as deuterium molecular gas targets and Li projectiles.

In the case of LiF target which is a large band gap insulator, one often approximates the electronic structure of the target ${ }^{6} \mathrm{Li}\left({ }^{7} \mathrm{Li}\right)$ state by the ${ }^{6} \mathrm{Li}^{+}\left({ }^{7} \mathrm{Li}^{+}\right)$with only two innermost electrons. Thus for all three reactions one expects the screening potential in the adiabatic limit $U_{e}^{(A D)}=$ $371.8-198.2 \sim 174 \mathrm{eV}$. Instead if one uses the ground state of the ${ }^{6} \mathrm{Li}\left({ }^{7} \mathrm{Li}\right)$ atom and of the bare deuteron target as the initial state, $U_{e}^{(A D)}=186 \mathrm{eV} 24$, which is given by the solid curve in Fig. 囵.

However one should be aware that the deuteron or hydrogen projectile plausibly moves with a bound electron in LiF solid insulator target [25. Under such an assumption we could estimate the screening potential $U_{e}^{(A D)}=$ $389.9-198.2 \sim 192 \mathrm{eV}$. In the case of molecular $\mathrm{D}_{2}$ or $\mathrm{H}_{2}$ gas targets, as well, we should consider the electron capture by the lithium projectile.

The bare S-factors for the same reaction have been extracted using an indirect method, the Trojan-Horse Method through the reaction ${ }^{6} \mathrm{Li}\left({ }^{6} \mathrm{Li}, \alpha \alpha\right){ }^{4} \mathrm{He}[8]$. The comparison between direct and the indirect methods gives the screening potential $U_{e}=320 \pm 50 \mathrm{eV}$. The corresponding enhancement factors are shown with the dash-dotted curve. The contrast between the direct measurement data and the theoretical estimation for the bare S-factor using the $R$-matrix theory gives $U_{e}=240 \mathrm{eV}$. It is shown with dotted line. The extracted $U_{e}$ with the two different methods are larger than the adiabatic limit.

We simulate the reactions ${ }^{6} \mathrm{Li}+d,{ }^{6} \mathrm{Li}+\mathrm{D},{ }^{7} \mathrm{Li}+p$ and ${ }^{7} \mathrm{Li}+\mathrm{H}$. In the figure [5 and 6) the open and closed squares show the enhancement factor for the reactions ${ }^{6} \mathrm{Li}+d$ and ${ }^{6} \mathrm{Li}+\mathrm{D},\left(\right.$ and ${ }^{7} \mathrm{Li}+p$ and $\left.{ }^{7} \mathrm{Li}+\mathrm{H}\right)$ respectively.

Again the average enhancement factors of the reaction ${ }^{6} \mathrm{Li}+\mathrm{D}\left({ }^{7} \mathrm{Li}+\mathrm{H}\right)$ are larger than those of the ${ }^{6} \mathrm{Li}+d\left({ }^{7} \mathrm{Li}+p\right)$. The enhancement factors of the reaction ${ }^{6} \mathrm{Li}+\mathrm{D}$ are in agreement with the extracted values using the $R$-matrix approach within the variances over all the events. The fit of the obtained average enhancement factors suggests the screening potentials $U_{e}=152.0 \pm 9.9 \mathrm{eV}$ for ${ }^{6} \mathrm{Li}+d$ and $U_{e}=214.4 \pm 18.5$ for ${ }^{6} \mathrm{Li}+\mathrm{D}$. The screening potential for the reaction ${ }^{6} \mathrm{Li}+d$ in our simulation does not exceed the adiabatic limit nor extracted values using the $R$-matrix theory and THM, but one for ${ }^{6} \mathrm{Li}+\mathrm{D}$ verges on the extracted values using the $R$-matrix approach. 


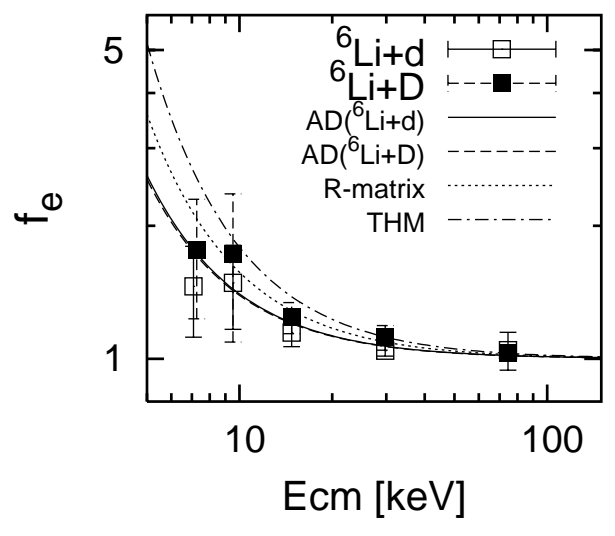

Fig. 5. same as Fig. 4 but for the reactions ${ }^{6} \mathrm{Li}+d$ and ${ }^{6} \mathrm{Li}+\mathrm{D}$.

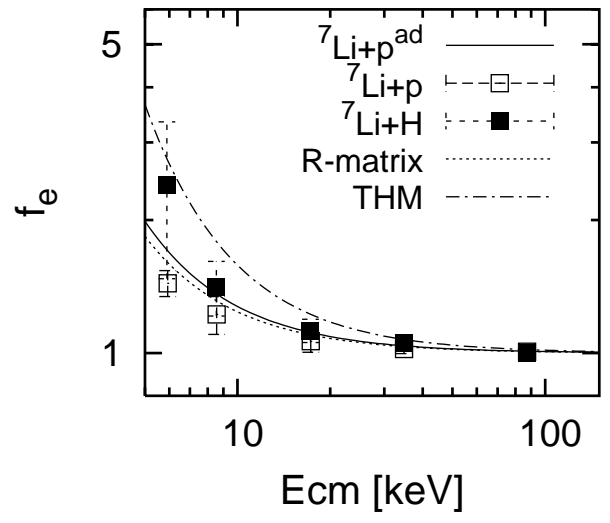

Fig. 6. same as Fig. 4 but for the reactions ${ }^{7} \mathrm{Li}+p$ and ${ }^{7} \mathrm{Li}+\mathrm{H}$.

\section{Summary}

We discussed the effect of the screening by the electrons in nuclear reactions at the astrophysical energies. We performed molecular dynamics simulations with constraints and imaginary time for the reactions $\mathrm{D}+d, \mathrm{D}+\mathrm{D},{ }^{3} \mathrm{He}+d$, ${ }^{3} \mathrm{He}+\mathrm{D},{ }^{6} \mathrm{Li}+d,{ }^{6} \mathrm{Li}+\mathrm{D},{ }^{7} \mathrm{Li}+p,{ }^{7} \mathrm{Li}+\mathrm{H}$. For all the reactions it is shown that both the average enhancement factors and their variances increase as the incident energy becomes lower. Using bare projectiles we obtained the average screening potential smaller than the value in the adiabatic limit for all reactions. It is because of the excitation or emission of several bound electrons during the reactions. The comparison between bare and atomic projectile cases for each reactions revealed that the electron capture of the projectile guides to larger enhancements. The derived enhancement factors in our simulation are in agreement with those extracted within the $R$-matrix approach including the variances over all the events.

We report also the results of the numerical experiments using polarized targets for the reaction $\mathrm{D}+\mathrm{d}$. Using $\mathrm{P}_{\perp}$ targets we obtained relatively large enhancements with small variances, instead $\mathrm{P}_{\|}$target gives large variances of the enhancement factors and relatively small averaged en- hancement factors. It is because with the $\mathrm{P}_{\|}$targets the force exerted from the electron to the relative motion of the nuclei is oscillational, in the direction of the beam axis, and the motion of the electron becomes often excited or unstable. It is the case where the chaoticity of the electron motion affects the tunneling probability and at the same time the enhancement factor of the cross section. This suggests that if one performs experiments at very low bombarding energies with polarized targets, the enhancement factor can be controlled by changing the polarization. The largest enhancement with targets polarized perpendicularly to the beam direction.

\section{References}

1. W.A.Lin and L.E. Ballentine, Phys. Rev. Lett. 65, 2927(1990); O. Bohigas, S. Tomosvic, and D. Ullumo. Phys. Rev. Lett., 65, 5(1990); A. Shudo, and K.S.Ikeda, Phys. Rev. Lett., 74, 682(1995).

2. S. Kimura, and A. Bonasera, Phys. Rev. Lett. 93 (2004) 262502; S. Kimura, and A. Bonasera, nucl-th/0504005 Nucl. Phys. A in press.

3. A. Krauss, et al., Nucl. Phys. A 467(1987) 273; S. Engstler et al., Phys. Lett. B 202 (1988) 179.

4. C. Rolfs, and E. Somorjai, Nucl. Instrum. Meth. B 99 (1995) 297.

5. F. C. Barker, Nucl. Phys. A 707 (2002) 277.

6. M. Junker, et al. Phys. Rev. C 57 (1998) 2700.

7. M. Lattuada, et al., Astrophys. J. 562 (2001) 1076 .

8. A. Musumarra, et al., Phys. Rev. C 64 (2001) 068801.

9. T. D. Shoppa, S. E. Koonin, K. Langanke, and R. Seki, Phys. Rev. C 48 (1993) 837.

10. S. Kimura, N. Takigawa, M. Abe, and D.M. Brink, Phys. Rev. C 67 (2003) 022801(R).

11. T. D. Shoppa, et al., Nucl. Phys. A 605 (1996) 387.

12. M. Papa, T. Maruyama, and A. Bonasera, Phys. Rev. C 64 (2001) 024612; S. Terranova, and A. Bonasera, Phys. Rev. C 70 (2004) 024906.

13. H. J. Assenbaum, and K. Langanke and C. Rolfs, Z. Phys. A 327(1987) 461.

14. D. D. Clayton, Principles of Stellar Evolution and Nucleosynthesis (University of Chicago Press, 1983) Chap. 4.

15. S. Kimura, and A. Bonasera, physics/0409008, Phys. Rev. A in press.

16. A. Bonasera, and V. N. Kondratyev, Phys. Lett. B 339 (1994) 207; T. Maruyama, A. Bonasera, and S. Chiba, Phys. Rev. C 63 (2001) 057601.

17. Y. Kato, N. Takigawa, nucl-th/0404075

18. F. Sattin, and L. Salasnich, Phys. Rev. E 59 (1999) 1246;

F. Sattin, and L. Salasnich, J. Phys. B, 29 (1996) L699.

19. K. Langanke, T.D.Shoppa, C.A.Barnes, and C.Rolfs, Phys. Lett. B 369 211(1996).

20. M. Aliotta, et al., Nucl.Phys.A 690, 790(2001).

21. M. La Cognata, et al., to be published in Nucl. Phys. A

22. T. Geyer and J. M. Rost, J. Phys. B: At. Mol. Opt. Phys. $36 \mathrm{~L} 107(2003)$. and references there in.

23. S. Engstler et al., Phys. Lett. B 202 (1988) 179.

24. L. Bracci, G. Fiorentini, V.S. Melezhik, G. Mezzorani, and P. Quarati, Nucl. Phys. A 513 (1990) 316.

25. K. Eder, et al., Phys. Rev. Lett. 79 4112(1997); P. Roncin, et al., Phys. Rev. Lett. 83 864(1999) 